\title{
LATTICE POINTS AND LIE GROUPS. II
}

\section{ROBERT S. CAHN(1)}

ABSTRACT. Let $C$ be the Ca simir operator on a compact, simple, simply connected Lie group $G$ of dimension $n$. The number of eigenvalues of $C$, counted with their multiplicities, of absolute value less than or equal to $t$ is asymptotic to $k t^{n / 2}, k$ a constant. This paper shows the error of this estimate to be $O\left(t^{2 b+a(a-1) /(a+1)}\right)$; where $a=$ rank of $G$ and $b=1 / 2(n-a)$.

Let $G$ be a compact, simple, simply connected Lie group and $B$ its Lie algebra. Following the terminology of Lattice points and Lie groups. I we have $\mathcal{F}$ a Cartan subalgebra of $\&, \mathcal{S}^{*}$ its dual, $($,$) the Killing form, R$ the roots of \&, $a_{M}=\operatorname{dim} \mathcal{F}, b_{M}=1 / 2|R|$.

The Lie algebra of a Lie group is defined as the left or right invariant tangent vector fields. If we choose to consider them as left invariant then $U(\&)$, the universal enveloping algebra, becomes identified with the left invariant differential operators and $U(\&)$ acts on $C^{\infty}(G)$.

If $B$ is a simple Lie a lgebra, the Casimir operator $C$ is defined as $\sum_{i, j=1}^{a} g^{i j} X_{i} X_{j}$ where the $X_{i}$ form a base for and $g^{i j}$ are the elements of the matrix $\left(g_{i j}\right)^{-1}$ where $g_{i j}=\left(X_{i}, X_{j}\right)$. This definition is independent of the basis chosen, and since $G$ is compact, the matrix $\left(g_{i j}\right)$ represents a nondegenerate negative definite quadratic form. Thus $\left(g^{i j}\right)$ represents a negative definite form and the operator $C$ is elliptic. The asymptotic behavior of the eigenvalues of elliptic operators have been studied in great generality. If $D$ is a selfadjoint elliptic operator defined on a compact manifold and $E(D, T)$ equals the number of eigenvalues of $D$ less than or equal to $T$ counted $w$ ith their multiplicities then $E(D, T) \sim$ $k T^{n / m}$ where $n$ is the dimension of the manifold and $m$ is the degree of $D$ [1]. For open bounded subsets of $R^{n}$ and $D$ with smooth coefficients the error for this estimate is $O\left(T^{n / m-1 / 2 m+\epsilon}\right)$ for any $\epsilon>0$ [2]. For compact subsets of manifolds with $D$ again smooth, the error is $O\left(T^{(n-1) / m}\right)$ [4]. However, for $C$ the Casimir operator of a compact, simple, simply connected Lie group a better estimate may be made.

Received by the editors March 18, 1971 and, in revised form, October 27, 1972. AMS (MOS) subject classifications (1970). Primary 22C05, $35 \mathrm{P} 20$.

Key words and phrases. Compact simple Lie group, lattice points, elliptic operator, Poisson summation formula.

(1) The results in this paper constitute part of the author's thesis. 
The left-regular representation of $G$ is a direct sum of all finite-dimensional irreducible representations of $G$, each occurring with multiplicity equal to its dimension, and the functions in the representation spaces may be assumed to be in $C^{\infty}(G)$. Also, $C$, regarded as a differential operator, commutes with each representation, so each representation space is an eigenspace of $C$. Moreover, as we shall see, the corresponding eigenvalue depends only on the representation, and can be explicitly calculated in terms of the corresponding dominant weight $\lambda$. Finally, if $G$ is simply connected, there is a bijection between representations of $G$ and $B$. Thus, using the terminology of $[1], E(C, T)$ or for brevity $E(T)$, equals

$$
\sum_{\Lambda \geq \delta, c_{\Lambda} \leq T} f_{\Theta}^{2}(\Lambda)
$$

where $f_{\circlearrowleft}(\Lambda)$ is the Weyl dimension polynomial and $\pi_{\lambda}(C)=c_{\Lambda} \operatorname{Id}, \Lambda=\lambda+\delta$. Since $f_{\Theta}=0$ along the walls of the positive Weyl chamber $P$, this sum equals

$$
\sum_{\mathbf{\Lambda} \geq 0, c_{\mathbf{\Lambda}} \leq T} f_{\Theta}^{2}(\Lambda) .
$$

To clarify this sum we wish to compute $c_{\Lambda}$. To do this fix a Cartan subalgebra $\mathscr{H} \subset \mathbb{B}$ and $\alpha_{1}, \cdots, \alpha_{a}$ simple roots. Each $\mathbb{F}_{a}$ is one-dimensional so pick $X_{a} \in \mathbb{B}_{a}$ for all roots $a$ and normalize the $X_{a}$ so that $\left(X_{a}, X_{-a}\right)=1$. Then $H_{a}=\left[X_{a}, X_{-a}\right]$. Pick the basis for $X_{i}=H_{a_{i}}, i=1, \ldots, a$, and $X_{i}=X_{a}$, $i=a+1, \cdots, n$. Then $g_{i j} \neq 0 \Rightarrow i, j \leq a$ or that $X_{i}=X_{a}$ and $X_{j}=X_{-a}$, and in this case $g_{i j}=1$. From this it is seen

$$
\begin{aligned}
C & =\sum_{i, j=1}^{a} g^{i j} H_{a_{i}} H_{\alpha_{j}}+\sum_{a} X_{a} X_{-a}=\sum_{i, j=1}^{a} g^{i j} H_{\alpha_{i}} H_{\alpha_{j}}+\sum_{\alpha>0}\left(X_{a} X_{-a}+X_{-a} X_{\alpha}\right) \\
& =\sum_{i, j=1}^{a} g^{i j} H_{a_{i}} H_{\alpha_{j}}+\sum_{\alpha>0} 2 X_{-a} X_{a}+\sum_{\alpha>0} H_{a} .
\end{aligned}
$$

Pick $v \in V_{\boldsymbol{\lambda}}$. Since $\lambda$ is the dominant weight of $\pi_{\boldsymbol{\lambda}}, \pi_{\boldsymbol{\lambda}}\left(X_{\alpha}\right) \nu=0, \alpha>0$, so

$$
\begin{aligned}
\pi_{\lambda}(C) v & =\left\{\sum_{i, j=1}^{a} g^{i j} \lambda\left(H_{a_{i}}\right) \lambda\left(H_{a_{j}}\right)\right\} v+\sum_{\alpha>0} \lambda\left(H_{\alpha}\right) v=\left(|\lambda|^{2}+\sum_{\alpha>0} \lambda\left(H_{\alpha}\right)\right) v \\
& =\left(|\lambda|^{2}+2 \cdot \frac{1}{2} \sum_{\alpha>0}(\lambda, \alpha)+|\delta|^{2}-|\delta|^{2}\right) v=\left(|\Lambda|^{2}-|\delta|^{2}\right) v .
\end{aligned}
$$

Thus $c_{\boldsymbol{\Lambda}}=|\Lambda|^{2}-|\delta|^{2}$ and

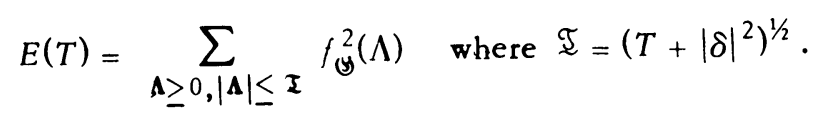

The sum runs over the lattice points in a solid angle of the $a$-ball in $B(\mathfrak{T})$ in $\mathscr{S}_{2}^{*}$.

We would like, for analytical purposes, to have the sum extend over the whole of $B(\mathfrak{T})$ so we build up $f_{(S)}^{2}$ by the action of the Weyl group. The Weyl 
group $W$ and its properties are found in [8, Chapter 5]. It is generated by reflections so that $W$ maps $B(\mathfrak{T})$ into itself and $W$ is simply transitive on the Weyl chambers of $\mathcal{F}^{*}\left[5\right.$, p. 242]. From this it follows that $f_{\Theta}^{2}$ is invariant under $W$. Let $\mu$ and $\mu^{\prime}$ be two sets of simple roots. Then there exists a unique $w \in W$ such that $w \mu=\mu^{\prime}$. Let

$$
f_{\mathcal{S}, \mu}(\Lambda)=c \prod_{a>0 \text { relative to } \mu}(\Lambda, \alpha)
$$

where $\Lambda>0$ relative to $\mu$.

where $\Lambda>0$ relative to $\mu^{\prime}$.

$$
f_{\Theta, \mu^{\prime}}(\Lambda)=c \prod_{\alpha>0 \text { relative to } \mu^{\prime}}(\Lambda, \alpha)
$$

$$
\begin{aligned}
\dot{f}_{\Theta, \mu}^{2}(\Lambda) & =\left(c \prod_{a>0 \text { relative to } \mu}(\Lambda, a)\right)^{2} \\
& =\left(c(-1)^{\text {length of } w} \underset{a>0 \text { relative to } \mu}{ }(w \Lambda, w a)\right)^{2} \\
& =\left(\begin{array}{l}
c \\
a>0 \text { relative to } \mu^{\prime}
\end{array}\right.
\end{aligned}
$$

Thus $f^{2}$ may be regarded as a single function on $B(\mathfrak{T})$ and

$$
\sum_{\mathbf{\Lambda}>0, \boldsymbol{\Lambda} \in B(\mathfrak{I})} f_{\mathcal{M}}^{2}(\Lambda)=\frac{1}{|W|} \sum_{\mathbf{\Lambda} \in B(\mathfrak{I})} f_{M}^{2}(\Lambda) .
$$

Normalizing Lebesgue measure such that the volume of the fundamental domain of

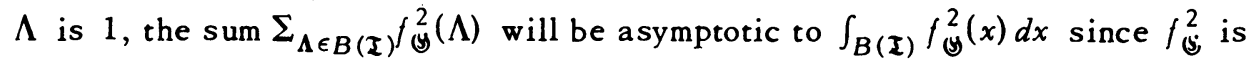
homogeneous. Taking the coordinate change $y=x / \mathcal{T}$ then $d y=d x / \mathcal{T}^{a}$ and this integral equals

$$
\int_{B(1)} f^{2}(\mathfrak{Y} y) \mathfrak{T}^{a} d y
$$

and, since $f$ is homogeneous of degree $b$,

$$
=\int_{B(1)} f_{\mathbb{S}}^{2}(y) \mathscr{\tau}^{2 b} \mathfrak{g}^{a} d y
$$

and, since $\operatorname{dim} G=2 b+a$,

$$
=\mathfrak{I} \operatorname{dim} G \int_{B(1)} f_{\mathscr{S}}^{2}(y) d y .
$$

The latter integral is a constant which we will call $d_{\circlearrowleft}$. Therefore

$$
E(T)=d_{(M)} /|W| \mathfrak{g} \operatorname{dim} G+o(\mathfrak{g} \operatorname{dim} G) .
$$

The purpose of this paper will be to estimate $R(T)=|| W\left|E(T)-d_{(} \mathfrak{F}^{\text {dim }} G\right|$.

The most elementary way of estimating $R(T)$ is to note that $R(T)$ is less 
than $f_{\mathscr{S}}^{2}(y)$ integrated over a shell of width $2 a^{1 / 2}$ around the boundary of $B(\mathfrak{T})$. This, in turn, is less than the maximum of $f_{\mathscr{S}}^{2}(y)$ in this region, $k \mathfrak{T}^{2 b}$, times the volume, which is $k^{\prime} \mathfrak{T}^{a-1}$. Therefore $R(T)$ is $O\left(\mathfrak{T}^{\mathrm{dim}} G^{-1}\right)$. However, for better results we will have to turn to analytic techniques using the Poisson summation formula. The methods are adaptations of those of Randol and his work will be referred to in the following pages.

Theorem. If $G$ is a compact, simple, simply connected Lie group then $R(T)$ is $O\left(\mathfrak{S}^{2 b+a}(a-1) / a+1\right)$.

Proof. Let $g_{T}(x)=f_{\circlearrowleft}^{2}(x) \chi_{B(\mathfrak{I})}(x)$ where $\chi_{B(\mathfrak{I})}(x)$ is the characteristic function of the ball of radius $\mathfrak{T}$. Since $f_{\Theta}^{2}(x)$ is analytic $g_{T}(x)$ would be of rapid decrease were there not a discontinuity on the boundary of $B(\mathfrak{T})$. To remove this we convolve $g_{T}(x)$ with a smoothing function. Let $\delta_{1}(x): \mathbf{R}^{a} \rightarrow \mathbf{R}^{+}$with support on $B(1)$ and $\int_{\mathrm{R} a} \delta_{1}(x) d x=1$. Define $\delta_{\epsilon}(x)=\left(1 / \epsilon^{a}\right) \delta_{1}(x / \epsilon)$. Then $\delta_{\epsilon}$ has support on $B(1)$ and $\int_{\mathrm{R} a} \delta_{\epsilon}(x) d x=1$. Define

$$
b_{T}(x)=g_{T} * \delta_{\epsilon}(x)
$$

Then $b_{T}$ is $C^{\infty}$ and of compact support. If $L$ is the lattice $\mathbf{Z} \lambda_{1} \oplus \cdots \oplus \mathbf{Z} \lambda_{n}$ and $\hat{L}$ is the dual lattice in $\hat{\mathbf{R}}^{a}=\mathbf{R}^{a}$ we have

$$
\sum_{n \in L} b_{T}(n)=\sum_{n \in \hat{L}} \hat{b}_{T}(n)=\sum_{n \in \hat{L}} \hat{\delta}_{\epsilon}(n) \hat{g}_{T}(n)=\hat{\delta}_{\epsilon}(0) \hat{g}_{T}(0)+\sum_{n \in \hat{L}}^{\prime}-\hat{\delta}_{\epsilon}^{\prime}(n) \hat{g}_{T}(n)
$$

where $\Sigma^{\prime}$ means summation over all lattice points except the origin. Now $\hat{\delta}_{\epsilon}(0)=$ $\int_{\mathrm{R} a} \delta_{\epsilon}(x) d x=1$ and

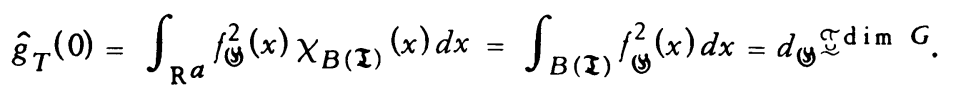

Thus $\Sigma_{n \in L} b_{T}(n)=d_{\circlearrowleft} \mathfrak{g}$ dim $G+\Sigma^{\prime} \hat{\delta}_{\epsilon}(n) \hat{g}_{T}(n)$. By the triangle inequality

$$
\begin{aligned}
& R(T) \leq\left|\sum_{n \in L} b_{T}(n)-d_{\circlearrowleft} \mathfrak{F}^{\operatorname{dim} G}\right|+|| W\left|E(T)-\sum_{n \in L} b_{T}(n)\right| \\
& =\sum_{n \in \hat{L}}\left|\hat{\delta}_{\epsilon}(n) \hat{g}_{T}(n)\right|+\sum_{n \in L}\left|b_{T}(n)-g_{T}(n)\right| .
\end{aligned}
$$

We wish to prove both sums $O\left(\mathfrak{T}^{2 b+a(a-1) / a+1}\right)$.

We first estimate the second sum. If $|n|>\mathfrak{T}+\epsilon$ then $g_{T}(n)=b_{T}(n)=0$. If $|n|<\mathfrak{T}-\epsilon$ then

$$
\left|b_{T}(n)-g_{T}(n)\right| \leq \underset{y \in B(\epsilon)}{\operatorname{Max}}\left|g_{T}(n+y)-g_{T}(n)\right|=\left|\operatorname{Grad} f_{\Theta}^{2}\left(x^{\prime}\right)\right| \epsilon
$$

for some $x^{\prime}$ such that $d\left(x^{\prime}, n\right) \leq \epsilon$. Since $f_{\mathbb{S}}(x)$ is homogeneous of degree $b$, $\left|\operatorname{Grad} f^{2}\left(x^{\prime}\right)\right| \leq k \mathfrak{T}^{2 b-1}$, so that $\left|b_{T}(n)-g_{T}(n)\right| \leq k \mathfrak{S}^{2 b-1} \epsilon$. There are less than $k^{\prime} \mathfrak{T}^{a}$ lattice points in $B(\mathfrak{T}-\epsilon)$ so $\Sigma_{n \in B(\mathfrak{I}-\epsilon)}\left|b_{T}(n)-g_{T}(n)\right|$ is $O\left(\mathfrak{T}^{2 b+a-1} \epsilon\right)$. 
If $\mathfrak{T}+\epsilon \geq|n| \geq \mathfrak{T}-\epsilon$ then $\left|b_{T}(n)-g_{T}(n)\right| \leq k \mathfrak{T}^{2 b}$. By [3] the number of lattice points in $\left.B(\mathfrak{T})=c \mathfrak{T}^{a}+O\left(\mathfrak{T}^{a}(a-1) / a+1\right)\right)$. Thus the number of lattice points in $B(\mathfrak{T}+\epsilon)-B(\mathfrak{W}-\epsilon)$ is $O\left(\mathfrak{T}^{a-1} \epsilon\right)$ if $\epsilon \geq \mathfrak{T}-(a-1) /(a+1)$. With this restriction on $\epsilon$, $\Sigma_{n \in B(\mathfrak{I}+\epsilon)-B\left(\mathfrak{I}-\epsilon^{\prime}\right)}\left|b_{T}(n)-g_{T}(n)\right|$ is $O\left(\mathfrak{I}^{2 b+a-1} \epsilon\right)$. This leaves

$$
\sum_{n \in \hat{L}}^{\prime}\left|\hat{\delta}_{\epsilon}(n) \hat{g}_{T}(n)\right|
$$

to analyze.

We begin by noting

$$
\hat{\delta}_{\epsilon}(x)=\epsilon^{-a} \widehat{\delta_{1}(x / \epsilon)}=\hat{\delta}_{1}(\epsilon x)
$$

Since $\delta_{1}$ is of rapid decrease in $x, \hat{\delta}_{\epsilon}$ is of rapid decrease in $\epsilon x$. The tricky point is the behavior of $\hat{g}_{T}(x)$. By the Riemann-Lebesgue lemma $\hat{g}_{T}(x) \rightarrow 0$ as $|x| \rightarrow \infty$ but to estimate (2) we need much better est imates for $\hat{g}_{T}(x)$.

Lemma. Let $f \in C^{\infty}\left(B^{a}(p)\right)$. Then $f \cdot \chi_{B(p)}(z)$ is $O\left(|z|^{-(a+1) / 2}\right)$.

Proof. If $f=1$ this is well known [3]. By definition

$$
\widehat{f \cdot \chi_{B(p)}}(z)=\int_{B(p)} e^{2 \pi i(x, z)} f(x) d x .
$$

We would like to transform this into a boundary integral by use of the divergence theorem. We wish to show that there exists a vector field $\left(g_{i}(x, z)\right)$ such that

$$
f(x) e^{2 \pi i(x, z)}=\operatorname{div}_{x}\left(\left(e^{2 \pi i(x, z)} / 2 \pi i|z|\right) \cdot\left(g_{i}(x, z)\right)\right)
$$

where the $g_{i}(x, z)$ and their first $m$ derivatives can be bounded in terms of $f$ and its first $m$ derivatives independent of $z$. Expanding the right side of (3):

$$
\begin{aligned}
\operatorname{div}_{x}\left(\left(e^{2 \pi i(x, z)} / 2 \pi i|z|\right)\left(g_{i}(x, z)\right)\right) & \\
& =\left(e^{2 \pi i(x, z) / 2 \pi i|z|)}\left[\sum_{i=1}^{a}\left(2 \pi i z_{i} g_{i}(x, z)\right)+\partial g_{i} / \partial x_{i}(x, z)\right] .\right.
\end{aligned}
$$

The latter expression equals $f(x) e^{2 \pi i(x, z)}$ if the two equations

$$
\begin{aligned}
& \sum_{i=1}^{a} \partial g_{i} / \partial x_{i}(x, z)=0, \\
& \sum_{i=1}^{a} \beta_{i} g_{i}(x, z)=f(x), \text { where } \beta_{i}=z_{i} /|z|,
\end{aligned}
$$

can be solved simultaneously. Such a solution of class $C^{\infty}$ is found in [7, Lemma 3]. Thus 


$$
\int_{B(p)} f(x) e^{2 \pi i(x, z)} d x=-\frac{1}{2 \pi i \mid z} \int_{\partial B(p)} e^{2 \pi i(y, z)} g_{1}(y, z) d y
$$

where $g_{1}(y, z)=(g(y, z), n(y)) ; n(y)$ is the unit normal at $y$. The lemma now follows from the estimate for Fourier transforms on p. 766 of [6].

We wish to use the lemma to estimate (2).

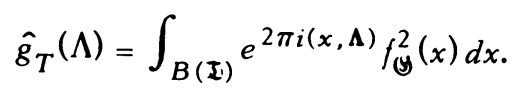

Let $x=\mathfrak{T}_{y}$ then $d x=\mathfrak{T}^{a} d y$ and

$$
\begin{aligned}
& \hat{g}_{T}(\Lambda)=\int_{B(1)} e^{2 \pi i\left(\mathfrak{I}_{y}, \Lambda\right)} f_{(\zeta)}^{2}\left(\mathfrak{F}_{y}\right) \mathfrak{I}^{a} d y
\end{aligned}
$$

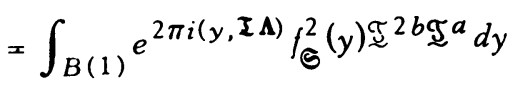

$$
\begin{aligned}
& =\mathfrak{\mho}^{2 b+a} \hat{g}_{1}(\mathfrak{T} \Lambda) .
\end{aligned}
$$

By the lemma $\hat{g}_{1}(\mathfrak{T} \Lambda) \leq k|\mathfrak{I} \Lambda|^{-(a+1 / 2)}$ so

$$
\left|\hat{g}_{T}(\Lambda)\right| \leq k \mathfrak{\varkappa}^{2 b+(a-1) / 2}|\Lambda|^{-(a+1) / 2}
$$

As was mentioned before, $\hat{\delta}_{\epsilon}(\Lambda)$ is of rapid decrease in $\epsilon \Lambda$ so there exists $k_{1}$ such that

$$
\hat{\delta}_{\epsilon}(\Lambda) \leq k_{1} /(1+|\epsilon \Lambda|)^{a} \text { for any } \Lambda \neq 0
$$

Then

$$
\sum_{\boldsymbol{\Lambda} \epsilon \hat{L}}^{\prime}\left|\hat{\delta}_{\epsilon}(\Lambda) \hat{g}_{T}(\Lambda)\right| \leq k_{2} \mathfrak{\Im}^{2 b+(a-1) / 2} \sum_{\boldsymbol{\Lambda} \epsilon \hat{L}}^{\prime}|\Lambda|^{-(a+1) / 2}(1+|\epsilon \Lambda|)^{-a}
$$

We now choose $\epsilon=\mathfrak{I}^{-(a+1) /(a+1)}$. To compute $\Sigma_{n \epsilon \hat{L}^{\prime}}|\Lambda|^{-(a+1) / 2}(1+|\epsilon \Lambda|)^{-a}$ we divide it into two sums

$$
\sum_{|\Lambda| \leq 1 / \epsilon}^{\prime}|\Lambda|^{-(a+1) / 2}(1+|\epsilon \Lambda|)^{-a}+\sum_{|\Lambda|>1 / \epsilon}|\Lambda|^{-(a+1) / 2}(1+|\epsilon \Lambda|)^{-a} .
$$

Comparing these sums to integrals

$$
\sum_{|\Lambda| \leq 1 / \epsilon}^{\prime}|\Lambda|^{-(a+1) / 2}(1+|\epsilon \Lambda|)^{-a} \leq k \int_{1}^{1 / \epsilon} x^{-(a+1) / 2} \cdot x^{a-1} d x+k^{\prime}
$$

where $k$ accounts for the volume of the parallelopiped spanned by the $\lambda_{i}$ and $k^{\prime}$ for the sum of all $\Lambda$ such that $|\Lambda|<2$. Thus

$$
\begin{aligned}
& \quad \sum_{|\mathbf{\Lambda}| \leq 1 / \epsilon}^{\prime}|\Lambda|^{-(a+1) / 2}(1+|\epsilon \Lambda|)^{-a} \leq k \int_{1}^{1 / \epsilon} x^{(a-3) / 2} d x+k^{\prime} \\
& \quad=2 k /\left.(a-1) x^{(a-1) / 2}\right|_{1} ^{\mathfrak{I}(a-1) /(a+1)}+\kappa^{\prime}=2 k /(a-1) \mathfrak{T}^{(a-1)^{2 / 2(a+1)}}+k^{\prime} .
\end{aligned}
$$

Similarly 


$$
\begin{aligned}
& \sum_{|\Lambda|>1 / \epsilon}|\Lambda|^{-(a+1) / 2}(1+|\epsilon \Lambda|)^{-a} \leq k \int_{1 / \epsilon}^{\infty} x^{-(a+1) / 2}(\epsilon x)^{-a} x^{a-1} d x+k_{0}^{\prime} \\
& \quad=\frac{k}{\epsilon^{a}} \int_{1 / \epsilon}^{\infty} x^{-(a+3) / 2} d x+k_{0}^{\prime}=k^{\prime} \epsilon^{(a+1) / 2-a}+k_{0}^{\prime}=k^{\prime} \mathscr{T}^{(a-1)^{2 / 2(a+1)}+k_{0}^{\prime} .}
\end{aligned}
$$

Thus

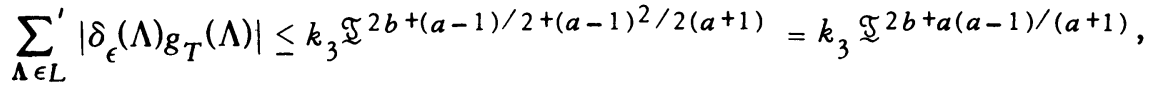

$$
\begin{aligned}
& \sum_{\Lambda \in B(\mathfrak{I})}\left|b_{T}(\Lambda)-g_{T}(\Lambda)\right| \leq k \mathfrak{T}^{2 b+a-1} \epsilon \\
& =k \mathfrak{I}^{2 b+a-1-(a-1) /(a+1)}=k \mathfrak{S}^{2 b+a(a-1) /(a+1)}
\end{aligned}
$$

so $R(T)$ is $O\left(\mathfrak{S}^{2 b+a(a-1) /(a+1)}\right)$.

\section{BIBLIOGRAPHY}

1. R. Beals, Global asymptotic estimates for elliptic spectral functions and eigenvalues, Bull. Amer. Math. Soc. 74 (1968), 358-360. MR 36 \#4172.

2. - On eigenvalue distributions for elliptic operators without smooth coefficients, Bull. Amer. Math. Soc. 72 (1966), 701-705. MR 33 \#4449.

3. C. S. Herz, On the number of lattice points in a convex set, Amer. J. Math 84 (1962), 126-133. MR 25 \#3015.

4. L. Hörmander, The spectral function of an elliptic operator, Acta Math. 121 (1968), 193-218.

5. N. Jacobson, Lie algebras, Interscience Tracts in Pure and App. Math., no. 10, Interscience, New York, 1962. MR 26 \# 1345.

6. W. Littman, Fourier transforms of surface-carried measures and differentiability of surface averages, Bull. Amer. Math. Soc. 69 (1963), 766-770. MR 27 \#5086.

7. B. Randol, A lattice-point problem, Trans. Amer. Math. Soc. 121 (1966), 257-268. MR $34 \# 1291$.

8. J.-P. Serre, Algèbres de Lie semi-simples complexes, Benjamin, New York, 1966. MR $35 \# 6721$. 33124

DEPARTMENT OF MATHEMATICS, UNIVERSITY OF MIAMI, CORAL GABLES, FLORIDA 